\title{
Current densities in density-functional theory
}

\author{
Elliott H. Lieb* \\ Princeton University, Departments of Physics and Mathematics, Jadwin Hall, Princeton, New Jersey 08544, USA \\ Robert Schrader ${ }^{\dagger}$ \\ Institut für Theoretische Physik, Freie Universität Berlin, Arnimallee 14, D-14195 Berlin, Germany
}

(Received 20 August 2013; published 26 September 2013)

\begin{abstract}
It is well known that any given density $\rho(\mathbf{x})$ can be realized by a determinantal wave function for $N$ particles. The question addressed here is whether any given density $\rho(\mathbf{x})$ and current density $\mathbf{j}(\mathbf{x})$ can be simultaneously realized by a (finite kinetic energy) determinantal wave function. In case the velocity field $\mathbf{v}(\mathbf{x})=\mathbf{j}(\mathbf{x}) / \rho(\mathbf{x})$ is curlfree, we provide a solution for all $N$, and we provide an explicit upper bound for the energy. If the velocity field is not curl-free, there is a finite energy solution for all $N \geqslant 4$, but we do not provide an explicit energy bound in this case. For $N=2$ we provide an example of a non-curl-free velocity field for which there is a solution and an example for which there is no solution. The case $N=3$ with a non-curl-free velocity field is left open.
\end{abstract}

DOI: 10.1103/PhysRevA.88.032516

PACS number(s): $31.15 . \mathrm{E}-$

\section{INTRODUCTION}

A question that arose in the early stages of densityfunctional theory is whether, given the one-body density $\rho(\mathbf{x})$ of an $N$-body system of fermions, there exists an $N$-body wave function (with finite kinetic energy) whose reduced one-body density equals the given one. More particularly, can this be accomplished with a determinantal wave function (under the obvious, necessary assumption, which will be made throughout, that $\nabla \sqrt{\rho}$ is square integrable).

This article provides a proof of the existence of a fermionic $N$-body determinantal state with a given one-body density $\rho(\mathbf{x})$ and a given one-body current density $\mathbf{j}(\mathbf{x})$ provided the velocity field $\mathbf{v}(\mathbf{x})=\mathbf{j}(\mathbf{x}) / \rho(\mathbf{x})$ is curl-free. When $N \geqslant 4$, we prove the existence of solutions even if the velocity field is not curl-free, like when there are vortices, for example. The proof is much more complicated in this case. To avoid dwelling on unenlightening points of mathematical rigor, definitions of function spaces, smoothness, and other technical questions are left to the reader. We do assume the obvious requirement that the support of $\mathbf{j}$ is contained in the support of $\rho$ and, for simplicity, that $\mathbf{j}, \rho$, and $\mathbf{v}$ are differentiable. Actually we assume that $\rho$ and $\mathbf{v}$ are given and thus we let the current be defined as $\mathbf{j}=\rho \mathbf{v}$.

In addition we provide a solution for an example with $N=$ 2 in which $\mathbf{v}(\mathbf{x})$ is not curl-free, which implies that "curlfreeness" is not a necessary condition for finding a solution when $N=2$. Again in the $N=2$ case, an example is provided for which no solution exists. This is contrary to a claim made without proof in Ref. [1] [see the discussion preceding relation (A1) there] that there always is a solution. The same claim was made in Ref. [2] [see the sentence containing relation (54) there]. Presently, it remains an open problem whether solutions always exist when $N=3$.

To avoid possible confusion, we emphasize that we are discussing only the existence of determinantal functions with the stated density and current; this state is not required to be the

\footnotetext{
*lieb@princeton.edu

${ }^{\dagger}$ schrader@physik.fu-berlin.de
}

ground state of any Hamiltonian. We also note that we consider only spinless (i.e., spin-polarized) particles here; spin can be included but it is an unnecessary complication here.

\section{STATEMENT OF THE PROBLEM}

Notation. In our units, $\hbar=1$ and the particle mass and particle charge are $m=1 / 2$ and $-e=-1$. Vectors are denoted by boldface. The density associated with a one-particle function $\phi$ is given by $\rho(\mathbf{x})=|\phi(\mathbf{x})|^{2}$. The current density is given by

$\mathbf{j}_{\phi}(\mathbf{x})=\frac{1}{2 i}\left[\phi^{*}(\mathbf{x}) \nabla \phi(\mathbf{x})-\phi(\mathbf{x}) \nabla \phi^{*}(\mathbf{x})\right]:=\frac{1}{2 i} \phi^{*}(\mathbf{x}) \overleftrightarrow{\nabla} \phi(\mathbf{x})$

which also defines the symbol $\overleftrightarrow{\nabla}$. This current is often called the paramagnetic current. Clearly, $\int_{\mathbb{R}^{3}} \nabla \cdot \mathbf{j}(\mathbf{x}) d \mathbf{x}=0$ by Green's theorem. The actual physical current, in the presence of the magnetic vector potential $\mathbf{A}(\mathbf{x})$, equals $\mathbf{j}(\mathbf{x})+\mathbf{A}(\mathbf{x}) \rho(\mathbf{x})$. Since $\rho(\mathbf{x})$ and $\mathbf{A}(\mathbf{x})$ are regarded as given, the additional $\mathbf{A} \rho$ term is thereby fixed and can be ignored for our considerations.

A fermionic $N$-body wave function $\psi\left(\mathbf{x}_{1}, \mathbf{x}_{2}, \ldots, \mathbf{x}_{N}\right)$ is totally antisymmetric and normalized, i.e.,

$\|\psi\|^{2}=\int_{\mathbb{R}^{3 N}}\left|\psi\left(\mathbf{x}_{1}, \mathbf{x}_{2}, \ldots, \mathbf{x}_{N}\right)\right|^{2} d \mathbf{x}_{1} d \mathbf{x}_{2} \cdots d \mathbf{x}_{N}=1$.

As stated above, spin variables could, but will not be, included in our discussion. The associated kinetic energy is defined as

$$
T(\psi)=\sum_{i=1}^{N} \int_{\mathbb{R}^{3 N}}\left|\nabla_{\mathbf{x}_{i}} \psi\left(\mathbf{x}_{1}, \mathbf{x}_{2}, \ldots, \mathbf{x}_{N}\right)\right|^{2} d \mathbf{x}_{1} d \mathbf{x}_{2} \cdots d \mathbf{x}_{N} .
$$

To this function $\psi$ we associate the one-body density

$$
\rho_{\psi}(\mathbf{x})=N \int_{\mathbb{R}^{3(N-1)}}\left|\psi\left(\mathbf{x}, \mathbf{x}_{2}, \ldots, \mathbf{x}_{N}\right)\right|^{2} d \mathbf{x}_{2} \cdots d \mathbf{x}_{N},
$$

such that by $(2.1)$

$$
\int_{\mathbb{R}^{3}} \rho_{\psi}(\mathbf{x}) d \mathbf{x}=N
$$


The associated current density is

$$
\begin{aligned}
\mathbf{j}(\mathbf{x})= & \frac{N}{2 i} \int_{\mathbb{R}^{3(N-1)}} \psi^{\star}\left(\mathbf{x}, \mathbf{x}_{2}, \ldots, \mathbf{x}_{N}\right) \overleftrightarrow{\nabla_{\mathbf{x}}} \psi^{\star}\left(\mathbf{x}, \mathbf{x}_{2}, \ldots, \mathbf{x}_{N}\right) \\
& \times d \mathbf{x}_{2} \cdots d \mathbf{x}_{N} .
\end{aligned}
$$

When $\phi_{1}(\mathbf{x}), \ldots, \phi_{N}(\mathbf{x})$ are orthonormal functions on $\mathbb{R}^{3}$, the $N$-body determinantal state

$$
\psi\left(\mathbf{x}_{1}, \cdots, \mathbf{x}_{N}\right)=(N !)^{-1 / 2} \operatorname{det}\left\{\phi_{k}\left(\mathbf{x}_{i}\right)\right\}_{1 \leqslant i, k \leqslant N}
$$

is normalized and

$$
\begin{aligned}
& \rho_{\psi}(\mathbf{x})=\sum_{k=1}^{N}\left|\phi_{k}(\mathbf{x})\right|^{2}=\sum_{k=1}^{N} \rho_{k}(\mathbf{x}), \\
& \mathbf{j}_{\psi}(\mathbf{x})=\frac{1}{2 i} \sum_{k=1}^{N} \phi_{k}^{\star}(\mathbf{x}) \overleftrightarrow{\nabla} \phi_{k}(\mathbf{x})=\sum_{k=1}^{N} \mathbf{j}_{k}(\mathbf{x}), \\
& T(\psi)=\sum_{k=1}^{N} \int_{\mathbb{R}^{3}}\left|\nabla \phi_{k}(\mathbf{x})\right|^{2} d \mathbf{x} .
\end{aligned}
$$

We look for one-body functions that can be written as

$$
\phi_{k}(\mathbf{x})=\rho_{k}(\mathbf{x})^{1 / 2} e^{i \chi_{k}(\mathbf{x})},
$$

with single-valued phase functions $\chi_{k}$ and with the orthonormality property

$$
\begin{aligned}
\left\langle\phi_{l}, \phi_{k}\right\rangle & =\int_{\mathbb{R}^{3}} \rho_{k}(\mathbf{x})^{1 / 2} \rho_{l}(\mathbf{x})^{1 / 2} \exp \left\{i\left[\chi_{l}(\mathbf{x})-\chi_{k}(\mathbf{x})\right]\right\} d \mathbf{x} \\
& =\delta_{k l} .
\end{aligned}
$$

Our condition (2.8) should be noted. We are restricting ourselves to functions with a well-defined global phase. For example, the function $\psi(\mathbf{x})=\left(x^{1}+i x^{2}\right) e^{-|x|^{2}}$ is a real analytic function that solves the problem for a smooth $\mathbf{j}$ and $\rho$, whose velocity field has a curl (a $\delta$ function), yet it has no global phase function. The fact that we can solve the problem for $N \geqslant 4$ with functions having a well-defined phase is, therefore, of some interest.

The second equation in Eq. (2.7) takes the form

$$
\mathbf{j}(\mathbf{x})=\sum_{k=1}^{N} \mathbf{j}_{k}(\mathbf{x})=\sum_{k=1}^{N} \rho_{k}(\mathbf{x}) \nabla \chi_{k}(\mathbf{x}) .
$$

Our finite kinetic energy condition means that each component of the vector field $\nabla \phi_{k}(\mathbf{x})$ is square integrable.

Finally, we define two energies: the kinetic energy of a density $\rho(\mathbf{x})$,

$$
E(\rho)=\int_{\mathbb{R}^{3}}\left|\nabla \rho(\mathbf{x})^{1 / 2}\right|^{2} d \mathbf{x},
$$

and the kinetic energy of a current density $\mathbf{j}$,

$$
E(\mathbf{j} ; \rho)=\int_{R^{3}} \frac{1}{\rho(\mathbf{x})}|\mathbf{j}(\mathbf{x})|^{2} d \mathbf{x}=\int_{\mathbb{R}^{3}} \rho(\mathbf{x})|\mathbf{v}(\mathbf{x})|^{2} d \mathbf{x},
$$

with the velocity field

$$
\mathbf{v}(\mathbf{x})=\frac{1}{\rho(\mathbf{x})} \mathbf{j}(\mathbf{x}) .
$$

One quickly checks that, for a determinantal function,

$$
T(\psi)=\sum_{k=1}^{N}\left[E\left(\rho_{k}\right)+E\left(\mathbf{j}_{k} ; \rho_{k}\right)\right] .
$$

This identity is the motivation for introducing the kinetic energy associated with a density and with a current density.

Now we can formulate the current-density problem.

The current-density problem. Given a density $\rho(\mathbf{x})$ with $\int \rho(\mathbf{x}) d \mathbf{x}=N$ and a current density $\mathbf{j}(\mathbf{x})$, satisfying $\int_{\mathbb{R}^{3}} \nabla$. $\mathbf{j}(\mathbf{x}) d \mathbf{x}=0$, is there an $N$-body determinantal state $\psi$, with functions as in Eq. (2.8), and with $\rho_{\psi}(\mathbf{x})=\rho(\mathbf{x})$ and $\mathbf{j}_{\psi}(\mathbf{x})=$ $\mathbf{j}(\mathbf{x})$ ? Suppose, in addition, $E(\rho)+E(\mathbf{j} ; \rho)<\infty$. Can this state be chosen to satisfy $T(\psi)<\infty$ ? If so, what bound can be placed on $T(\psi)$ ?

For a physical motivation of this problem, see Ref. [2]. For a previous discussion of this problem in the one-dimensional case, see Refs. [1,2].

We recall a result for the case in which the density $\rho(\mathbf{x})$ alone is considered; that is, no $\mathbf{j}(\mathbf{x})$ is prescribed, and hence the second condition is merely $E(\rho)<\infty$. This was solved affirmatively, independently, and by the same method in Refs. [3,4]. The solution happens, incidentally, to have the property that $\mathbf{j}=0$.

The following bound appears in Ref. [4]:

Suppose $E(\rho)<\infty$. Then there is an $N$-body determinantal state $\psi$ satisfying $\rho_{\psi}=\rho$ and

$$
T(\psi) \leqslant(4 \pi)^{2} N^{2} E(\rho) .
$$

Subsequently, Zumbach improved $N^{2}$ to $N^{2 / 3}$ [5].

\section{SOLUTION OF THE CURRENT-DENSITY PROBLEM FOR A CURL-FREE VELOCITY FIELD}

In this section we solve the problem for arbitrary $N \geqslant 1$, when the velocity field is curl free, $\nabla \times \mathbf{v}=0$. See Theorem 1. In Sec. IV we drop this condition and are able to solve the problem when $N \geqslant 4$. First, we recall the well-known solution $[3,4]$ to the familiar problem of finding $\psi$ which solves $\rho_{\psi}=\rho$ for given $\rho$. Write $\mathbf{x}=\left(x^{1}, x^{2}, x^{3}\right)$ and define for $-\infty<x^{3}<$ $\infty$

$$
f\left(x^{3}\right)=\frac{2 \pi}{N} \int_{-\infty}^{\infty} \int_{-\infty}^{+\infty} \int_{-\infty}^{x^{3}} \rho(s, t, u) d s d t d u,
$$

which is monotone increasing from 0 to $2 \pi$. For given $N$ we introduce the set of $N$ numbers

$$
\mathcal{K}_{N}=\left\{-\frac{N-1}{2},-\frac{N-3}{2}, \ldots, \frac{N-3}{2}, \frac{N-1}{2}\right\},
$$

satisfying

$$
\sum_{k \in \mathcal{K}_{N}} k=0
$$

Set

$$
C_{N}=\frac{16 \pi^{2}}{N} \sum_{k \in \mathcal{K}_{N}} k^{2}
$$

Define

$$
\phi_{k}(\mathbf{x})=[\rho(\mathbf{x}) / N]^{1 / 2} \exp \left\{i k f\left(x^{3}\right)\right\}, \quad k \in \mathcal{K}_{N},
$$


and $\psi\left(\mathbf{x}_{1}, \ldots, \mathbf{x}_{N}\right)=(N !)^{-1 / 2} \operatorname{det} \phi_{k}\left(\mathbf{x}_{j}\right)$. Then $\rho_{k}(\mathbf{x})=$ $\rho(\mathbf{x}) / N$ and $\chi_{k}(\mathbf{x})=k f\left(x^{3}\right)$. These $N$ functions $\left\{\phi_{k}\right\}$ are orthonormal. The kinetic energy of the determinantal state $\psi$ has the bound given in Ref. [4]:

$$
T(\psi) \leqslant\left(1+C_{N}\right) E(\rho) .
$$

Since we establish a similar bound later, let us briefly recall the argument for (3.6). We start with (2.14), where the first sum on the right-hand side is

$$
\sum_{k=1}^{N} E\left(\rho_{k}\right)=E(\rho)
$$

For the second sum we have to compute $E\left(\mathbf{j}_{k} ; \rho_{k}\right)$ for these functions $\phi_{k}(\mathbf{x})$, and we have that

$$
\rho_{k}(\mathbf{x})\left|\nabla \chi_{k}(\mathbf{x})\right|^{2}=\frac{(2 \pi)^{2} \rho(\mathbf{x})}{N^{3}} k^{2} g\left(x^{3}\right)^{4},
$$

with the definition

$$
g(u)^{2}=\int_{-\infty}^{\infty} \int_{-\infty}^{\infty} \rho(s, t, u) d s d t=\frac{N}{2 \pi} \frac{d}{d u} f(u) .
$$

Hence

$$
\begin{aligned}
& \sum_{k \in \mathcal{K}_{N}} E\left(\mathbf{j}_{k} ; \rho_{k}\right) \\
& \quad=\int_{\mathbb{R}^{3}} \rho_{k}(\mathbf{x})\left|\nabla \chi_{k}(\mathbf{x})\right|^{2} d \mathbf{x} \\
& \quad=\frac{(2 \pi)^{2}}{N^{3}} \sum_{k \in \mathcal{K}_{N}} k^{2} \int_{-\infty}^{\infty} \int_{-\infty}^{\infty} \int_{-\infty}^{\infty} \rho(s, t, u) g(u)^{4} d s d t d u \\
& =\frac{(2 \pi)^{2}}{N^{3}} \sum_{k \in \mathcal{K}_{N}} k^{2} \int_{-\infty}^{\infty} g(u)^{6} d u .
\end{aligned}
$$

As shown in Ref. [4],

$$
\int_{-\infty}^{\infty} g(u)^{6} d u \leqslant 4 N^{2} E(\rho) .
$$

For the convenience of the reader we briefly recall its proof. Since

$$
g(u)^{2}=2 \int_{-\infty}^{u} g(v) \frac{d g(v)}{d v} d v
$$

holds, we conclude by the Schwarz inequality that for all $u$

$$
g(u)^{4} \leqslant 4 \int_{-\infty}^{\infty} g(v)^{2} d v \int_{-\infty}^{\infty}\left(\frac{d g(v)}{d v}\right)^{2} d v \equiv P .
$$

The first integral on the right-hand side equals $N$ by the normalization condition on $\rho$. Therefore we obtain the estimate

$$
\begin{aligned}
\int_{-\infty}^{\infty} g(u)^{6} d u & \leqslant P \int_{-\infty}^{\infty} g(u)^{2} d u \\
& =4 N^{2} \int_{-\infty}^{\infty}\left(\frac{d g(v)}{d v}\right)^{2} d v .
\end{aligned}
$$

To conclude the proof of (3.9) we must show that

$$
\int_{-\infty}^{\infty}\left(\frac{d g(v)}{d v}\right)^{2} d v \leqslant E(\rho)
$$

holds. To do this write

$\int_{-\infty}^{\infty}\left(\frac{d g(v)}{d v}\right)^{2} d v=\frac{1}{4} \int_{-\infty}^{\infty} \frac{\left(\int_{-\infty}^{\infty} \int_{-\infty}^{\infty} \frac{\partial}{\partial v} \rho(x, y, v) d x d y\right)^{2}}{\int_{-\infty}^{\infty} \int_{-\infty}^{\infty} \rho\left(x^{\prime}, y^{\prime}, v\right) d x^{\prime} d y^{\prime}} d v$

and then use

$$
\begin{aligned}
& \left(\int_{-\infty}^{\infty} \int_{-\infty}^{\infty} \frac{\partial}{\partial v} \rho(x, y, v) d x d y\right)^{2} \\
& =\left(\int_{-\infty}^{\infty} \int_{-\infty}^{\infty} 2 \sqrt{\rho(x, y, v)} \frac{\partial}{\partial v} \sqrt{\rho(x, y, v)} d x d y\right)^{2} \\
& \leqslant 4 \int_{-\infty}^{\infty} \int_{-\infty}^{\infty} \rho(x, y, v) d x d y \\
& \quad \times \int_{-\infty}^{\infty} \int_{-\infty}^{\infty}\left(\frac{\partial}{\partial v} \sqrt{\rho(x, y, v)}\right)^{2} d x d y
\end{aligned}
$$

by Schwarz's inequality. Insert this bound into the right-hand side of (3.13) thereby proving (3.12). We insert inequality (3.9) into (3.8) and perform the sum over $k$. Collecting terms yields (3.6).

Theorem 1. Assume $\mathbf{v}(\mathbf{x})=\nabla \tau(\mathbf{x})$ for some function $\tau$, i.e., $\mathbf{v}$ is curl-free. For given $N \geqslant 1$ let

$$
\chi_{k}(\mathbf{x})=\tau(\mathbf{x})+k f\left(x^{3}\right), \quad k \in \mathcal{K}_{N} .
$$

The revised functions

$$
\phi_{k}(\mathbf{x})=\sqrt{\frac{\rho(\mathbf{x})}{N}} \exp \left\{i\left[\tau(\mathbf{x})+k f\left(x^{3}\right)\right]\right\},
$$

with $f$ given by (3.1), form an orthonormal system. The determinantal state $\psi$,

$$
\begin{aligned}
& \psi\left(\mathbf{x}_{1}, \ldots, \mathbf{x}_{N}\right) \\
& =\frac{1}{N !{ }^{1 / 2} N^{N / 2}} \prod_{k=1}^{N}\left(\sqrt{\rho\left(x_{k}\right)} e^{i \tau\left(\mathbf{x}_{k}\right)}\right) \operatorname{det}\left\{e^{i k f\left(x_{j}^{3}\right)}\right\},
\end{aligned}
$$

satisfies $\rho_{\psi}(\mathbf{x})=\rho(\mathbf{x})$ and $\mathbf{j}_{\psi}(\mathbf{x})=\mathbf{j}(\mathbf{x})=\rho(\mathbf{x}) \mathbf{v}(\mathbf{x})$ with the energy bound

$$
T(\psi) \leqslant C_{N} E(\rho)+E(\mathbf{j} ; \rho) .
$$

Proof. Clearly, the relation $\mathbf{j}_{\psi}=\mathbf{j}=\rho \mathbf{v}$ follows from the fact that $\sum_{k \in \mathcal{K}_{N}} k=0$. The proof of the first part follows from [4]. So we only have to prove the estimate (3.18). By (3.15) we have

$$
\nabla \chi_{k}(\mathbf{x})=\nabla \tau(\mathbf{x})+k \nabla f\left(x^{3}\right)=\mathbf{v}(\mathbf{x})+\frac{2 \pi}{N} k g\left(x^{3}\right)^{2} \mathbf{e}_{3},
$$

where $\mathbf{e}_{3}=(0,0,1)$ is the unit vector in the 3 -direction.

Since $\sum_{k} k=0$, we have that

$$
\sum_{k}\left|\nabla \chi_{k}(\mathbf{x})\right|^{2}=N|\mathbf{v}(\mathbf{x})|^{2}+\frac{4 \pi^{2}}{N^{2}} g\left(x^{3}\right)^{4} \sum_{k} k^{2} ;
$$

i.e., the cross term vanishes. Combined with $\rho_{k}=\rho / N$ (and the fact that $\rho_{k}$ is independent of $k$ ) this gives the inequality

$$
\begin{aligned}
& \sum_{k} \int_{\mathbb{R}^{3}} \rho_{k}(\mathbf{x})\left|\nabla \chi_{k}(\mathbf{x})\right|^{2} d \mathbf{x} \\
& \quad \leqslant \frac{1}{N} E(\mathbf{j} ; \rho)+\frac{4 \pi^{2}}{N^{2}} \sum_{k} k^{2} \int_{-\infty}^{\infty} g\left(x^{3}\right)^{6} d x^{3} .
\end{aligned}
$$

Using (3.7) and summing over $k$ gives the bound (3.18). 
In summary, the curl-freeness of $\mathbf{v}$ is a sufficient condition for solving the current-density problem.

\section{SOLUTION OF THE CURRENT-DENSITY PROBLEM FOR A NON-CURL-FREE VELOCITY FIELD WHEN $\mathbf{N} \geqslant 4$}

This section is devoted to a proof of Theorem 2 .

Theorem 2. Given $\rho$ and $\mathbf{j}$, when $N \geqslant 4$ there is always a determinantal wave function $\psi$ with $\rho_{\psi}=\rho$ and $\mathbf{j}_{\psi}=\mathbf{j}$. Moreover, if in addition $E(\rho)<\infty$ and $E(\mathbf{j}, \rho)<\infty$ and if the curl $\mathbf{w}=\nabla \times \mathbf{v}$ of $\mathbf{v}=\mathbf{j} / \rho$ and its first-order derivatives satisfy the bounds

$$
\begin{aligned}
& \sup _{\mathbf{x} \in \mathbb{R}^{3}, j=1,2,3}\left[1+\left(x^{1}\right)^{2}\right]^{(1+\delta) / 2}\left[1+\left(x^{2}\right)^{2}\right]^{(1+\delta) / 2} \\
& \times\left[1+\left(x^{3}\right)^{2}\right]^{(1+\delta) / 2}\left|w_{j}(\mathbf{x})\right|<\infty \\
& \sup _{\mathbf{x} \in \mathbb{R}^{3}, i, j=1,2,3}\left[1+\left(x^{1}\right)^{2}\right]^{(1+\delta) / 2}\left[1+\left(x^{2}\right)^{2}\right]^{(1+\delta) / 2} \\
& \quad \times\left[1+\left(x^{3}\right)^{2}\right]^{(1+\delta) / 2}\left|\partial_{i} w_{j}(\mathbf{x})\right|<\infty
\end{aligned}
$$

for some $\delta>0$, then $T(\psi)<\infty$.

We conjecture that condition (4.1) can be considerably loosened. We have used the notation $\partial_{i}=\partial / \partial_{x^{i}}$. The proof will be split into several steps. (To avoid clutter we will sometimes omit the dependence on $\mathbf{x}$ from now on, when the meaning is clear. Recall that $\mathbf{x}=\left(x^{1}, x^{2}, x^{3}\right)$ and do not confuse $x^{2}$ with $|\mathbf{x}|^{2}$.)

Step 1: Construct the $\rho_{i}$. We do this in such a way that all $\rho_{i}$ for $i \geqslant 4$ are equal, while the $\rho_{i}$ for $1 \leqslant i \leqslant 4$ are different. The motivation for this is that, in the case where the velocity field is not curl-free, we cannot choose all $\rho_{i}$ to be equal to $\rho / N$. Indeed, such an ansatz would give

$$
\mathbf{v}(\mathbf{x})=\frac{1}{\rho(\mathbf{x})} \mathbf{j}(\mathbf{x})=\nabla \frac{1}{N} \sum_{i=1}^{N} \chi_{i}(\mathbf{x})
$$

by (2.10), which shows that $\operatorname{curl} \mathbf{v}=0$ and which is a contradiction. However, we may and do choose $N-3$ of them to be equal. Set

$$
\xi(x)=\frac{1}{m} \int_{-\infty}^{x} \frac{1}{\left(1+y^{2}\right)^{(1+\delta) / 2}} d y,
$$

with

$$
m=\int_{-\infty}^{\infty} \frac{1}{\left(1+y^{2}\right)^{(1+\delta) / 2}} d y,
$$

and where $\xi(x)$ is a continuous, strictly increasing function in $x$ with $\xi(-\infty)=0$ and $\xi(\infty)=1 . \delta$ is the $\delta$ in Eq. (4.1) if the curl $\mathbf{w}$ of $\mathbf{v}$ satisfies the bound (4.1) and is arbitrary $>0$ otherwise. Set $\rho_{i}=\eta_{i} \rho$, with

$$
\begin{aligned}
& \eta_{1}(\mathbf{x})=\frac{2}{N} \xi\left(x^{1}+\alpha\right) \\
& \eta_{2}(\mathbf{x})=\frac{2}{N-1} \xi\left(x^{1}+\beta\right)\left[1-\eta_{1}(\mathbf{x})\right] \\
& \eta_{3}(\mathbf{x})=\frac{2}{N-2} \xi\left(x^{2}+\gamma\right)\left[1-\eta_{1}(\mathbf{x})-\eta_{2}(\mathbf{x})\right], \\
& \left.\eta_{i}(\mathbf{x})=\frac{1}{N-3}\left[1-\eta_{1}(\mathbf{x})-\eta_{2}(\mathbf{x})-\eta_{3}(\mathbf{x})\right], \quad 4 \leqslant i \leqslant N .2\right)
\end{aligned}
$$

$\alpha, \beta$, and $\gamma$ are real and, for the moment, arbitrary.
Observe that $\eta_{1}$ and $\eta_{2}$ are functions of the first component $x^{1}$ of $\mathbf{x}$ only, while the $\eta_{j}$ for $j \geqslant 3$ depend on $x^{1}$ and $x^{2}$ but not on $x^{3}$. We claim $0 \leqslant 1-\eta_{1}(\mathbf{x})-\eta_{2}(\mathbf{x})$ and $0 \leqslant 1-\eta_{1}(\mathbf{x})-$ $\eta_{2}(\mathbf{x})-\eta_{3}(\mathbf{x})$ hold and thus $0 \leqslant \eta_{j}$ for all $1 \leqslant j \leqslant N$. Indeed, an easy calculation gives

$$
\begin{aligned}
1- & \eta_{1}(\mathbf{x})-\eta_{2}(\mathbf{x}) \\
= & {\left[1-\eta_{1}(\mathbf{x})\right]\left(1-\frac{2}{N-1} \xi\left(x^{1}+\beta\right)\right), } \\
1- & \eta_{1}(\mathbf{x})-\eta_{2}(\mathbf{x})-\eta_{3}(\mathbf{x}) \\
= & {\left[1-\eta_{1}(\mathbf{x})\right]\left(1-\frac{2}{N-1} \xi\left(x^{1}+\beta\right)\right) } \\
& \times\left(1-\frac{2}{N-2} \xi\left(x^{2}+\gamma\right)\right),
\end{aligned}
$$

and this combined with

$$
\begin{aligned}
& 1-\frac{2}{N-1} \xi\left(x^{1}+\beta\right) \geqslant \frac{N-3}{N-1}, \\
& 1-\frac{2}{N-2} \xi\left(x^{2}+\gamma\right) \geqslant \frac{N-4}{N-2}
\end{aligned}
$$

proves the claim.

As a consequence

$$
\begin{aligned}
& 0 \leqslant \rho_{1} \leqslant \frac{2}{N} \rho, \quad 0 \leqslant \rho_{2} \leqslant \frac{2}{N-1}\left(\rho-\rho_{1}\right), \\
& 0 \leqslant \rho_{3} \leqslant \frac{2}{N-2}\left(\rho-\rho_{1}-\rho_{2}\right), \\
& 0 \leqslant \rho_{i}=\frac{1}{N-3}\left(\rho-\rho_{1}-\rho_{2}-\rho_{3}\right), \\
& 4 \leqslant i \leqslant N, \quad \sum_{i=1}^{N} \rho_{i}=\rho .
\end{aligned}
$$

To fix $\alpha$, consider the function

$$
I(\alpha)=\int_{\mathbb{R}^{3}} \rho_{1}(\mathbf{x}) d \mathbf{x}=\frac{2}{N} \int_{\mathbb{R}^{3}} \xi\left(x^{1}+\alpha\right) \rho(\mathbf{x}) d \mathbf{x},
$$

which is continuous and monotonically strictly increasing in $\alpha$ (since $\xi$ has these properties). Since $\lim _{\alpha \rightarrow-\infty} I(\alpha)=0$ and $\lim _{\alpha \rightarrow+\infty} I(\alpha)=2$, these properties imply that there is a unique $\alpha$ such that $I(\alpha)=1$. We choose this value of $\alpha$ since it implies that $\int_{\mathbb{R}^{3}} \rho_{1} d \mathbf{x}=1$, as required. Having thus fixed $\alpha$, by the same argument and using the fact that $\int_{\mathbb{R}^{3}}\left(\rho-\rho_{1}\right) d \mathbf{x}=N-1$, we can fix $\beta$ uniquely such that also $\int_{\mathbb{R}^{3}} \rho_{2}=1$ is valid. Similarly, we can fix $\gamma$ such that also $\int_{\mathbb{R}^{3}} \rho_{3}=1$ is valid. But then we also have that, for $4 \leqslant i \leqslant N$,

$$
\begin{aligned}
\int_{\mathbf{x} \in \mathbb{R}^{3}} \rho_{i}(\mathbf{x}) d \mathbf{x} & =\frac{1}{N-3} \int_{\mathbb{R}^{3}}\left[\rho(\mathbf{x})-\rho_{1}(\mathbf{x})-\rho_{2}(\mathbf{x})-\rho_{3}(\mathbf{x})\right] d \mathbf{x} \\
& =1 .
\end{aligned}
$$

This completes the construction of all one-body densities $\rho_{i}, 1 \leqslant i \leqslant N$.

Step 2: Construction of phase functions $\chi_{i}$ satisfying (2.10). We postpone the implementation of the orthogonality to the remaining Steps 3-5. Given the $\rho_{i}$ and $\eta_{i}$ constructed in the previous step, Eq. (2.10) takes the equivalent form

$$
\sum_{i=1}^{3} \eta_{i} \nabla \chi_{i}+\eta_{4} \nabla\left(\sum_{i=4}^{N} \chi_{i}\right)=\mathbf{v} .
$$


Recall that we assumed $\mathbf{v}=\mathbf{j} / \rho$ to be well defined though $\rho$ may have zeros or even vanish in a region. As already mentioned in the Introduction the best way to avoid such problems is to assume $\rho$ and $\mathbf{v}$ to be given rather than $\rho$ and $\mathbf{j}$. The current $\mathbf{j}$ is then defined to equal $\rho \mathbf{v}$.

We introduce

$$
\tau(\mathbf{x})=\frac{1}{N-3} \sum_{i=4}^{N} \chi_{i}(\mathbf{x}) .
$$

Then, with the auxiliary quantities

$$
\widehat{\chi}_{i}=\chi_{i}-\tau, \quad i=1,2,3,
$$

Eq. (4.5) is equivalent to

$$
\nabla \tau=\mathbf{v}-\sum_{i=1}^{3} \eta_{i} \nabla \widehat{\chi}_{i}
$$

which in particular says that the right-hand side has to be curl-free. The strategy for determining the phase factors is as follows. We first determine the necessary form of the $\widehat{\chi}_{k}$ that makes the right-hand side of (4.8) curl free. Equation (4.8) then defines $\tau$ up to an uninteresting additive constant. In Step 3 the $\chi_{i}$ for $4 \leqslant i \leqslant N$ are determined in such a way that they satisfy (4.6) and such that the resulting wave functions $\phi_{i}=\rho_{i}^{1 / 2} \exp i \chi_{i}, 4 \leqslant i \leqslant N$, are orthogonal. For this we follow the strategy used in the proof of Theorem 1. Finally in Step 4 we determine the $\widehat{\chi}_{i}$ and hence the $\chi_{i}, 1 \leqslant i \leqslant 3$, via (4.7) such that all $\phi_{i}, 1 \leqslant i \leqslant N$, are pairwise orthogonal.

To implement these steps, we first take the curl of (4.8) and obtain the curl-freeness condition:

$$
\sum_{i=1}^{3} \nabla \eta_{i} \times \nabla \widehat{\chi}_{i}=\mathbf{w}=\operatorname{curl} \mathbf{v} .
$$

Using (4.2) we can write out (4.9) in components:

$$
\begin{aligned}
\partial_{2} \eta_{3} \partial_{3} \widehat{\chi}_{3} & =w_{1}, \\
-\partial_{1} \eta_{1} \partial_{3} \widehat{\chi}_{1}-\partial_{1} \eta_{2} \partial_{3} \widehat{\chi}_{2}-\partial_{1} \eta_{3} \partial_{3} \widehat{\chi}_{3} & =w_{2} \\
\partial_{1} \eta_{1} \partial_{2} \widehat{\chi}_{1}+\partial_{1} \eta_{2} \partial_{2} \widehat{\chi}_{2}+\partial_{1} \eta_{3} \partial_{2} \widehat{\chi}_{3}-\partial_{2} \eta_{3} \partial_{1} \widehat{\chi}_{3} & =w_{3}
\end{aligned}
$$

Recall that $\eta_{1}$ and $\eta_{2}$ depend on $x^{1}$ only, while $\eta_{3}$ depends on $x^{1}$ and $x^{2}$. As a consequence no partial derivatives of the form $\partial_{1} \widehat{\chi}_{1}$ or $\partial_{1} \widehat{\chi}_{2}$ appear in these equations. As preparation for the next step we calculate some of the partial derivatives of the $\eta$ 's. The inequalities

$$
\partial_{1} \eta_{1}(\mathbf{x})=\frac{2}{m N\left(1+\left(x^{1}+\alpha\right)^{2}\right)^{(1+\delta) / 2}}>0
$$

and

$$
\begin{aligned}
\partial_{2} \eta_{3}(\mathbf{x})= & \frac{2}{m(N-2)\left[1+\left(x^{2}+\gamma\right)^{2}\right]^{(1+\delta) / 2}} \\
& \times\left[1-\eta_{1}(\mathbf{x})-\eta_{2}(\mathbf{x})\right]>0
\end{aligned}
$$

are valid due to

$$
1-\eta_{1}(\mathbf{x})-\eta_{2}(\mathbf{x})>\frac{1}{6},
$$

an easy consequence of the definitions (4.2) of $\eta_{1}$ and $\eta_{2}$. In particular $\partial_{1} \eta_{1}$ and $\partial_{2} \eta_{3}$ never vanish.
Let $h_{1}, h_{2}$, and $h_{3}$ be arbitrary functions of $x^{1}$ only. Define

$$
\begin{aligned}
& \kappa_{1}(\mathbf{x})=\sum_{j=1}^{3} \kappa_{1, j}(\mathbf{x}), \quad \kappa_{2}(\mathbf{x})=0, \\
& \kappa_{3}(\mathbf{x})=\int_{0}^{x^{3}}\left(\frac{w_{1}}{\partial_{2} \eta_{3}}\right)\left(x^{1}, x^{2}, s\right) d s,
\end{aligned}
$$

with

$$
\begin{aligned}
\kappa_{1,1}(\mathbf{x})= & \frac{1}{\partial_{1} \eta_{1}\left(x^{1}\right)} \int_{0}^{x^{2}} w_{3}\left(x^{1}, s, x^{3}=0\right) d s \\
& -\frac{1}{\partial_{1} \eta_{1}\left(x^{1}\right)} \int_{0}^{x^{3}}\left(w_{2}+\frac{\partial_{1} \eta_{3}}{\partial_{2} \eta_{3}} w_{1}\right)\left(x^{1}, x^{2}, t\right) d t \\
\kappa_{1,2}(\mathbf{x})= & \frac{\partial_{1} h_{3}\left(x^{1}\right)}{\partial_{1} \eta_{1}\left(x^{1}\right)} \eta_{3}\left(x^{1}, x^{2}\right) \\
\kappa_{1,3}(\mathbf{x})= & -\frac{1}{\partial_{1} \eta_{1}\left(x^{1}\right)}\left[\left(\partial_{1} \eta_{2}\right) h_{2}\right]\left(x^{1}\right)
\end{aligned}
$$

In terms of these quantities the functions $\widehat{\chi}_{i}$ are defined as

$$
\widehat{\chi}_{i}=\kappa_{i}+h_{i}, \quad i=1,2,3 .
$$

Define

$$
\mathbf{u}=\mathbf{v}-\sum_{i=1}^{3} \eta_{i} \nabla \widehat{\chi}_{i}
$$

We have the following lemma.

Lemma 1. u is curl-free for arbitrary $h_{1}, h_{2}$, and $h_{3}$.

By what has been said so far, it suffices to check that (4.10) is satisfied. We give the proof in Appendix A. It is somewhat intricate and uses the fact that $\mathbf{w}$ has zero divergence. By this lemma $\mathbf{u}$ is a gradient field and we define $\tau$ to be the solution to the equation $\nabla \tau=\mathbf{u}$. $\tau$ is unique up to a constant and is therefore fixed uniquely by requiring it to vanish at the origin.

To sum up, we have determined $\widehat{\chi}_{1}, \widehat{\chi}_{2}, \widehat{\chi}_{3}$, and $\tau$ such that (4.8) holds. Finally we set

$$
\chi_{i}=\widehat{\chi}_{i}+\tau, \quad i=1,2,3 .
$$

Observe that by (4.13) and (4.14) all components of the curl $\mathbf{w}$ of $\mathbf{v}$ enter the definition of these three phase functions.

Step 3: Orthogonality for $4 \leqslant i \leqslant N$. We construct suitable phase functions $\widehat{\chi}_{i}, 4 \leqslant i \leqslant N$, to achieve the orthogonality of the corresponding $N-3$ one-body wave functions $\widehat{\phi}_{i}$

$$
\widehat{\phi}_{i}(x)=\rho_{i}(x)^{1 / 2} e^{i \widehat{\chi}_{i}(x)}, \quad 4 \leqslant i \leqslant N .
$$

To achieve this we refer to our discussion in Sec. III. Set

$$
\widehat{\rho}=\left(\rho-\rho_{1}-\rho_{2}-\rho_{3}\right)=(N-3) \rho_{4}
$$

such that $\int \widehat{\rho}(\mathbf{x}) d \mathbf{x}=(N-3)$, which puts us in a $(N-3)$ body context by which we may invoke the discussion of Sec. III. Indeed, the associated $N-3$-body current is

$$
\widehat{\mathbf{j}}=\sum_{i=4}^{N} \rho_{i} \nabla \chi_{i}=\frac{\widehat{\rho}}{N-3} \sum_{i=4}^{N} \nabla \chi_{i}=\widehat{\rho} \nabla \tau,
$$

and so the associated velocity field $1 / \widehat{\rho} \hat{\mathbf{j}}$ is a gradient field equal to $\mathbf{u}$ by the construction of $\tau$ (see the end of Step 3). 
With

$$
\widehat{f}\left(x^{3}\right)=\frac{2 \pi}{N-3} \int_{-\infty}^{+\infty} \int_{-\infty}^{+\infty} \int_{-\infty}^{x^{3}} \widehat{\rho}(s, t, u) d s d t d u
$$

and for $4 \leqslant i \leqslant N$, we adjust the notation in Eqs. (3.15) and (3.16) to the present situation and set

$$
\widehat{\chi}_{i}(\mathbf{x})=\left(i-4-\frac{N-4}{2}\right) \widehat{f}\left(x^{3}\right), \quad 4 \leqslant i \leqslant N .
$$

Observe that when $i$ runs through $4,5, \ldots, N$, then $i-4-$ $(N-4) / 2$ runs through the set $\mathcal{K}_{N-3}$ [see (3.2)]. By the arguments given in the proof of Theorem 1, the functions (4.18) form an orthonormal system of $N-3$ vectors.

Step 4: Orthogonality for $i=1,2,3$. Here we extend the orthonormal system (4.18) with the help of suitably chosen phases $\widehat{\chi}_{1}, \widehat{\chi}_{2}$, and $\widehat{\chi}_{3}$ and wave functions

$$
\widehat{\phi}_{k}(\mathbf{x})=\rho_{k}^{1 / 2}(\mathbf{x}) e^{i \widehat{\chi}_{k}(\mathbf{x})}, \quad 1 \leqslant k \leqslant 3,
$$

to an orthonormal system $\widehat{\phi}_{i}$ of $N$ vectors. This in turn means we have to find suitable functions $h_{1}, h_{2}$, and $h_{3}$ as introduced in Step 2. With this ansatz the scalar products, which we have to make vanish, can be written as

$$
\begin{aligned}
\left\langle\widehat{\phi}_{k}, \widehat{\phi}_{i}\right\rangle & =\int_{\mathbb{R}^{3}} e^{-i\left(\kappa_{k}+h_{k}-\widehat{x}_{i}\right)} \eta_{k}^{1 / 2} \eta_{i}^{1 / 2} \rho d \mathbf{x}, \\
\left\langle\widehat{\phi}_{k}, \widehat{\phi}_{l}\right\rangle & =\int_{\mathbb{R}^{3}} e^{-i\left(\kappa_{k}+h_{k}-\kappa_{l}-h_{l}\right)} \eta_{k}^{1 / 2} \eta_{l}^{1 / 2} \rho d \mathbf{x},
\end{aligned}
$$

for $1 \leqslant l, k \leqslant 3, l<k, 4 \leqslant i \leqslant N$. We invoke the following theorem in Ref. [6].

Theorem 3. Let $m \geqslant 1$ functions $\psi_{j} \in L^{1}\left(\mathbb{R}^{n}\right), 1 \leqslant j \leqslant$ $m$, be given. Then there exists a real, infinitely differentiable function $\chi(\mathbf{x})$ on $\mathbb{R}^{n}$, with bounded derivatives, such that

$$
\int_{\mathbb{R}^{n}} e^{-i \chi(\mathbf{x})} \psi_{j}(\mathbf{x}) d \mathbf{x}=0
$$

holds for all $1 \leqslant j \leqslant m$. The $\psi_{j}$ can be complex-valued.

The $\chi(\mathbf{x})$ constructed in Ref. [6] is a function of one variable only (which may be taken to be any one of the $x^{i}$ that one wishes) and vanishes outside a bounded set in that variable. Consequently, $\chi(\mathbf{x})$ has bounded derivatives. This implies that if the $\psi_{j}$ have finite kinetic energy [i.e., $\left.\nabla \psi \in L^{2}\left(\mathbb{R}^{n}\right)\right]$ then the functions $e^{-i \chi(\mathbf{x})} \psi_{j}(\mathbf{x})$ also have finite kinetic energy. Unfortunately, the theorem in Ref. [6] or the one in Ref. [7] does not tell us how large the kinetic energies of the $e^{-i \chi(\mathbf{x})} \psi_{j}(\mathbf{x})$ functions are, only that they are finite.

Theorem 3 is a generalization of the Hobby-Rice theorem [8] (see also [9]), according to which a piecewise constant $\chi(x)$ (equal to 0 or $\pi$ everywhere) exists with the property stated in Theorem 3. Such a $\chi$ would necessarily lead to infinite kinetic energy (because of the discontinuities) and would not be suitable for us. Theorem 3 tells us how to smooth out the discontinuities and is essential for us.

Theorem 3 can be used to orthogonalize any set of any $N$ functions, $f_{1}, \ldots, f_{N}$. It says that one can add a phase to $f_{2}$ so that $f_{1}$ and $f_{2}$ are orthogonal. Then one can add a phase to $f_{3}$ so that $f_{3}$ is orthogonal to $f_{1}$ and $f_{2}$. Finally, one can make $f_{N}$ orthogonal to $f_{1}, \ldots, f_{N-1}$.

In our case we have to proceed cautiously. We use the three undetermined functions $h_{1}, h_{2}$, and $h_{3}$ as phases, but the astute reader will notice that our functions already depend explicitly on $h_{3}$ and $h_{2}$ and might complain about lack of independence. In fact, only $\psi_{1}$ depends on $h_{3}$ and $h_{2}$. Thus, no problem arises if we do things in the right order: First we determine $h_{3}$ to make $\psi_{3}$ orthogonal to $\psi_{i}$ for $i \geqslant 4$. This fixes $h_{3}$. Then we fix $h_{2}$ similarly. Now $\psi_{1}$ is fixed and we are free to choose $h_{1}$ to complete the orthogonalization. The order is important!

We first consider the case $k=3$ in the first relation in Eq. (4.23). The aim is to find a suitable function $h_{3}$ depending on $x^{1}$ only. When we set

$$
\begin{aligned}
\psi_{i}^{(3)}\left(x^{1}\right) & =\int_{\left(x^{2}, x^{3}\right) \in \mathbb{R}^{2}} e^{-i\left(\kappa_{3}(\mathbf{x})-\widehat{\chi}_{i}(\mathbf{x})\right)} \eta_{3}^{1 / 2}(\mathbf{x}) \eta_{i}^{1 / 2}(\mathbf{x}) \rho(\mathbf{x}) d x^{2} d x^{3}, \\
4 & \leqslant i \leqslant N,
\end{aligned}
$$

an element of $L^{1}(\mathbb{R})$, we obtain

$$
\left\langle\widehat{\phi}_{3}, \widehat{\phi}_{i}\right\rangle=\int_{x^{1} \in \mathbb{R}} e^{-i h_{3}\left(x^{1}\right)} \psi_{i}^{(3)}\left(x^{1}\right) d x^{1}, \quad 4 \leqslant i \leqslant N .
$$

By the previous lemma we can find a continuously differentiable function $h_{3}$ such that all these expressions vanish. This choice of $h_{3}$ determines $\widehat{\chi}_{3}$. We turn to the case $k=2$ and introduce the following functions in $L^{1}(\mathbb{R})$ :

$$
\begin{aligned}
& \psi_{3}^{(2)}\left(x^{1}\right) \\
& =\int_{\left(x^{2}, x^{3}\right) \in \mathbb{R}^{2}} e^{-i\left[\kappa_{2}(\mathbf{x})-\kappa_{3}(\mathbf{x})-h_{3}\left(x^{1}\right)\right]} \eta_{2}^{1 / 2}(\mathbf{x}) \eta_{3}^{1 / 2}(\mathbf{x}) \rho(\mathbf{x}) d x^{2} d x^{3}, \\
& \psi_{i}^{(2)}\left(x^{1}\right) \\
& \quad=\int_{\left(x^{2}, x^{3}\right) \in \mathbb{R}^{2}} e^{-i\left[\kappa_{2}(\mathbf{x})-\widehat{\chi}_{i}(\mathbf{x})\right]} \eta_{2}^{1 / 2}(\mathbf{x}) \eta_{i}^{1 / 2}(\mathbf{x}) \rho(\mathbf{x}) d x^{2} d x^{3}, \\
& 4 \leqslant i \leqslant N,
\end{aligned}
$$

such that

$$
\left\langle\widehat{\phi}_{2}, \widehat{\phi}_{i}\right\rangle=\int_{\mathbb{R}} e^{-i h_{2}\left(x^{1}\right)} \psi_{i}^{(2)}\left(x^{1}\right) d x^{1}, \quad 3 \leqslant i \leqslant N .
$$

Again by the lemma there is a continuously differentiable function $h_{2}$ in the variable $x^{1}$ such that all these expressions vanish. This choice of $h_{2}$ determines $\widehat{\chi}_{2}$. Finally we turn to the case $k=1$. Set

$$
\begin{aligned}
& \begin{array}{l}
\psi_{2}^{(1)}\left(x^{1}\right) \\
=\int_{\left(x^{2}, x^{3}\right) \in \mathbb{R}^{2}} e^{-i\left[\kappa_{1}(\mathbf{x})-\widehat{\chi}_{2}(\mathbf{x})\right]} \eta_{1}(\mathbf{x})^{1 / 2} \eta_{2}(\mathbf{x})^{1 / 2} \rho(\mathbf{x}) d x^{2} d x^{3}, \\
\psi_{3}^{(1)}\left(x^{1}\right) \\
=\int_{\left(x^{2}, x^{3}\right) \in \mathbb{R}^{2}} e^{-i\left[\kappa_{1}(\mathbf{x})-\widehat{\chi}_{3}(\mathbf{x})\right]} \eta_{1}(\mathbf{x})^{1 / 2} \eta_{3}(\mathbf{x})^{1 / 2} \rho(\mathbf{x}) d x^{2} d x^{3}, \\
\psi_{i}^{(1)}\left(x^{1}\right) \\
\quad=\int_{\left(x^{2}, x^{3}\right) \in \mathbb{R}^{2}} e^{-i\left[\kappa_{1}(\mathbf{x})-\widehat{\chi}_{i}(x)\right]} \eta_{1}^{1 / 2}(\mathbf{x}) \eta_{i}^{1 / 2}(\mathbf{x}) \rho(\mathbf{x}) d x^{2} d x^{3},
\end{array} \\
& 4 \leqslant i \leqslant N,
\end{aligned}
$$

which again are elements of $L^{1}(\mathbb{R})$. Observe that $\kappa_{1}$ is known since $h_{3}$ and $h_{2}$ have been determined [see (4.13) and (4.14)]. By construction

$$
\left\langle\widehat{\phi}_{1}, \widehat{\phi}_{i}\right\rangle=\int_{\mathbb{R}} e^{-i h_{1}\left(x^{1}\right)} \psi_{i}^{(1)}\left(x^{1}\right) d x^{1}, \quad 2 \leqslant i \leqslant N,
$$

holds. We use the lemma a final time to find a function $h_{1}$ such that all these expressions vanish. 
To sum up, the $\widehat{\phi}_{i}$ for all $1 \leqslant i \leqslant N$ form an orthonormal system. Then

$$
\phi_{i}(\mathbf{x})=\widehat{\phi}_{i}(\mathbf{x}) e^{i \tau(\mathbf{x})}=\rho_{i}^{1 / 2}(\mathbf{x}) e^{i \chi_{i}(\mathbf{x})}, \quad 1 \leqslant i \leqslant N .
$$

are also orthonormal. By construction (2.10) holds, and the proof of the first part of Theorem 2 is finished. It remains to prove $T(\psi)<\infty$ when (4.1) holds. For this we take recourse to (2.14). Since

$$
\nabla \sqrt{\rho_{i}}=\nabla \sqrt{\eta_{i} \rho}=\left(\nabla \sqrt{\eta_{i}}\right) \sqrt{\rho}+\eta_{i} \nabla \sqrt{\rho}
$$

holds, by using the definition (4.2) one can easily check that each $\nabla \sqrt{\eta_{i}}$ is bounded. Since $\sqrt{\rho},|\nabla \sqrt{\rho}| \in L^{2}\left(\mathbb{R}^{3}\right)$ we conclude $\left|\nabla \sqrt{\rho_{i}}\right| \in L^{2}\left(\mathbb{R}^{3}\right)$; that is, $E\left(\rho_{i}\right)<\infty$ for all $i$. To estimate $E\left(\mathbf{j}_{i}, \rho_{i}\right)$ we proceed as follows. Since $\chi_{i}=\tau+\widehat{\chi}_{i}$ and $0 \leqslant \rho_{i} \leqslant \rho$,

$$
\int_{\mathbb{R}^{3}} \rho_{i}\left|\nabla \chi_{i}\right|^{2} d \mathbf{x} \leqslant 2 \int_{\mathbb{R}^{3}} \rho|\nabla \tau|^{2} d \mathbf{x}+2 \int_{\mathbb{R}^{3}} \rho\left|\nabla \widehat{\chi}_{i}\right|^{2} d \mathbf{x} .
$$

First we consider the case $i \geqslant 4$. Then the second term on the right-hand side is finite by the choice of $\widehat{\chi}_{i}$ and the discussion in Sec. III. By the definition of $\tau$ and relation (4.16),

$$
|\nabla \tau|^{2} \leqslant 8|\mathbf{v}|^{2}+8 \sum_{i=1}^{3}\left|\nabla \widehat{\chi}_{i}\right|^{2} .
$$

Since

$$
\int_{\mathbb{R}^{3}} \rho|\mathbf{v}|^{2} d \mathbf{x}=E(\mathbf{j}, \rho)<\infty,
$$

by assumption, we are done if we can show that

$$
\int_{\mathbb{R}^{3}} \rho\left|\nabla \widehat{\chi}_{i}\right|^{2} d \mathbf{x}<\infty
$$

holds for all $i=1,2,3$. Then incidentally the right-hand side of (4.25) is finite for all $1 \leqslant i \leqslant N$. By (4.15),

$$
\begin{gathered}
\int_{\mathbb{R}^{3}} \rho\left|\nabla \widehat{\chi}_{i}\right|^{2} d \mathbf{x} \leqslant 2 \int_{\mathbb{R}^{3}} \rho\left|\nabla \kappa_{i}\right|^{2} d \mathbf{x}+2 \int_{\mathbb{R}^{3}} \rho\left|\nabla h_{i}\right|^{2} d \mathbf{x}, \\
i=1,2,3 .
\end{gathered}
$$

The second term on the right-hand side is finite by the choice of the $h_{i}$, Theorem 3, and the comment thereafter. As for the first term, the case $i=2$ is trivial since $\kappa_{2}=0$. In Appendix B we prove Lemma 2.

Lemma 2. The functions $\left|\nabla \kappa_{i}\right|$ for $i=1,3$ are bounded.

Given this lemma the first integral on the right-hand side of (4.28) is also finite, thus completing the proof of Theorem 2.

\section{THE CASE $N=2, \nabla \times v \neq 0$}

In this section we discuss the case of two particles, $N=2$. Surprisingly, we have not been able to provide conditions that are both necessary and sufficient for a solution of the problem to exist. Of course curl-freeness of the velocity field is sufficient but not necessary as the first example shows. Conversely the second example provides a (non-curl-free) velocity field, for which there is no solution.

\section{A. Solution to an example with $N=\mathbf{2}$ and $\nabla \times \mathbf{v} \neq 0$.}

Let $\mathbf{c} \neq 0$ be a fixed vector and consider

$$
\rho(\mathbf{x})=2 \pi^{-3 / 2} e^{-|\mathbf{x}|^{2}}, \quad \mathbf{j}(\mathbf{x})=\pi^{3 / 2}(\mathbf{c} \times \mathbf{x}) e^{-|\mathbf{x}|^{2}},
$$

with the resulting velocity field

$$
\mathbf{v}(\mathbf{x})=\frac{1}{2}(\mathbf{c} \times \mathbf{x}),
$$

which is not curl-free. The normalization $\int_{\mathbb{R}^{3}} \rho(\mathbf{x}) d \mathbf{x}=2$ holds, $\nabla \cdot \mathbf{j}(\mathbf{x})=0$, and both $E(\rho)$ and $E(\mathbf{j} ; \rho)$ are finite. We consider the case where $\mathbf{c}=(0,0,1)$; a general $\mathbf{c}$ may be discussed similarly.

Assume there are $\rho_{1}>0, \rho_{2}>0, \chi_{1}$, and $\chi_{2}$, with $\rho_{1}+$ $\rho_{2}=\rho$, which are solutions to the equation

$$
\rho_{1} \nabla \chi_{1}+\rho_{2} \nabla \chi_{2}=\mathbf{j}
$$

subject to the condition

$$
\int_{\mathbb{R}^{3}} \rho_{j}(\mathbf{x})^{1 / 2} \rho_{k}(\mathbf{x})^{1 / 2} e^{i\left[\chi_{k}(\mathbf{x})-\chi_{j}(\mathbf{x})\right]} d \mathbf{x}=\delta_{j k} .
$$

Introduce $\eta_{k}(\mathbf{x})=\rho_{k}(\mathbf{x}) / \rho(\mathbf{x})$ which satisfies $0 \leqslant \eta_{k} \leqslant$ $1, \eta_{1}+\eta_{2}=1$. So we may rewrite (5.3) as

$$
\eta_{1} \nabla \chi_{1}+\eta_{2} \nabla \chi_{2}=\mathbf{v},
$$

or equivalently

$$
\eta_{1} \nabla\left(\chi_{1}-\chi_{2}\right)+\nabla \chi_{2}=\mathbf{v}
$$

and (5.4) as

$$
\int_{\mathbb{R}^{3}} \eta_{j}(x)^{1 / 2} \eta_{k}(\mathbf{x})^{1 / 2} e^{i\left[\chi_{k}(\mathbf{x})-\chi_{\mathbf{j}}(\mathbf{x})\right]} \rho(\mathbf{x}) d \mathbf{x}=\delta_{j k} .
$$

We take the curl of (5.5) and use $\nabla \eta_{2}(\mathbf{x})=-\nabla \eta_{1}(\mathbf{x})$, a consequence of the relation $\eta_{1}(\mathbf{x})+\eta_{2}(\mathbf{x})=1$. This gives

$$
\nabla \eta_{1}(\mathbf{x}) \times \nabla \widehat{\chi}(\mathbf{x})=\nabla \times \mathbf{v}(\mathbf{x})=\mathbf{c},
$$

with $\widehat{\chi}=\chi_{1}-\chi_{2}$, and is valid for all $\mathbf{x} \in \mathbb{R}^{3}$. As a consequence of (5.8) the vector fields $\nabla \eta_{1}(\mathbf{x})$ and $\nabla \widehat{\chi}(\mathbf{x})$ are never parallel and in particular never vanishing. In addition we conclude that they are orthogonal to $\mathbf{c}$.

We define

$$
\begin{aligned}
& \eta_{1}(\mathbf{x})=\frac{1}{2}\left(1+\tanh x^{1}\right), \\
& \widehat{\chi}(\mathbf{x})=2 x^{2} \cosh ^{2} x^{1}+h\left(x^{1}\right),
\end{aligned}
$$

where for the moment $h$ is an arbitrary function of $x^{1}$ alone. In particular

$$
\eta_{2}(\mathbf{x})=\frac{1}{2}\left(1-\tanh x^{1}\right)
$$

and $0 \leqslant \eta_{j} \leqslant 1$ is satisfied. Also by construction

$$
\nabla \times\left(\eta_{1} \nabla \widehat{\chi}-\mathbf{v}\right)=\mathbf{c} ;
$$

i.e., (5.8) is satisfied. But this implies there is a solution $\chi_{2}$ to (5.6). More explicitly

$$
\begin{aligned}
\chi_{2}(\mathbf{x})= & -x^{2}\left[\left(1+\tanh x^{1}\right) \cosh ^{2} x^{1}-x^{1}\right] \\
& -\frac{1}{2} \int_{0}^{x^{1}}(1+\tanh y) \frac{d}{d y} h(y) d y+\text { const. }
\end{aligned}
$$


where $h(y)$ is undetermined as yet. $\chi_{1}$ is of course given as $\widehat{\chi}-\chi_{2}$. Moreover, since tanh is odd,

$$
\int_{\mathbb{R}^{3}} \eta_{1} \rho d \mathbf{x}=\int_{\mathbb{R}^{3}} \eta_{2} \rho d \mathbf{x}=\frac{1}{2} \int_{\mathbb{R}^{3}} \rho d x=1 .
$$

Thus (5.4) is satisfied for $j=k=1,2$ for any choice of $h$. To determine $h$, we inspect the remaining condition $(j=2, k=$ 1) in Eq. (5.4), which we write in the form

$$
\begin{aligned}
\int_{R^{3}} \eta_{1} \eta_{2} \rho e^{i \widehat{x}} d \mathbf{x}= & \frac{1}{2 \pi^{3 / 2}} \int_{\mathbb{R}^{3}} \sqrt{1-\tanh ^{2} x^{1}} \\
& \times \mathrm{e}^{-\left(x^{1}\right)^{2}-\left(x^{2}\right)^{2}-\left(x^{3}\right)^{2}} e^{i\left[2 x^{2} \cosh ^{2} x^{1}+h\left(x^{1}\right)\right]} d \mathbf{x} \\
= & 0
\end{aligned}
$$

Set

$$
\begin{aligned}
g\left(x^{1}\right)= & \frac{1}{2 \pi^{3 / 2}} \sqrt{1-\tanh ^{2} x^{1}} e^{-\left(x^{1}\right)^{2}} \\
& \times \int_{\left(x^{2}, x^{3}\right) \in \mathbb{R}^{2}} e^{-\left(x^{2}\right)^{2}-\left(x^{3}\right)^{2}} e^{i 2 x^{2} \cosh ^{2} x^{1}} d x^{2} d x^{3} \\
= & \frac{1}{2 \pi^{1 / 2}} \sqrt{1-\tanh ^{2} x^{1}} e^{-\left(x^{1}\right)^{2}} e^{-\cosh ^{4} x^{1}}
\end{aligned}
$$

which is integrable and positive for all $x^{1}$. Condition (5.13) takes the form

$$
\int_{-\infty}^{\infty} g\left(x^{1}\right) e^{i h\left(x^{1}\right)} d x^{1}=0
$$

Set

$$
a=\int_{-\infty}^{+\infty} g(y) d y>0 .
$$

Then the choice

$$
h\left(x^{1}\right)=\frac{2 \pi}{a} \int_{-\infty}^{x^{1}} g(y) d y,
$$

with $h(-\infty)=0$ and $h(\infty)=2 \pi$ gives

$$
\begin{aligned}
\int_{-\infty}^{\infty} g\left(x^{1}\right) e^{i h\left(x^{1}\right)} d x^{1} & =\frac{a}{2 \pi i} \int_{-\infty}^{\infty} \frac{d}{d x^{1}} e^{i h\left(x^{1}\right)} d x^{1} \\
& =\frac{a}{2 \pi i}\left(e^{i h(\infty)}-\mathrm{e}^{i h(-\infty)}\right)=0 .
\end{aligned}
$$

By inserting this solution for $h$ into (5.11), all quantities are determined. We claim it gives a solution $\psi$ for which $T(\psi)<$ $\infty$. For the proof we use the identity (2.14). First $E\left(\rho_{1}\right)<\infty$ and $E\left(\rho_{2}\right)<\infty$ is an easy consequence of $E(\rho)<\infty$ and the choice of $\eta_{1}$ and $\eta_{2}$. An easy calulation gives the bound

$$
\left|\nabla \chi_{2}(\mathbf{x})\right| \leqslant 2\left|x^{2} \sinh 2 x^{1}\right|+2 \cosh ^{2} x^{1}+\left|x^{1}\right|+\left|\frac{\partial h\left(x^{1}\right)}{\partial x^{1}}\right|,
$$

but

$$
\frac{\partial h\left(x^{1}\right)}{\partial x^{1}}=\frac{2 \pi}{a} g\left(x^{1}\right)
$$

decreases strongly as $x^{1} \rightarrow \pm \infty$. Since $\rho_{2}<\rho$, and thanks to the Gaussian form of $\rho$, we therefore obtain

$$
E\left(\mathbf{j}_{2} ; \rho_{2}\right)=\int_{\mathbb{R}^{3}} \rho_{2}\left|\nabla \chi_{2}\right|^{2} d \mathbf{x}<\infty .
$$

As for $E\left(\mathbf{j}_{1} ; \rho_{1}\right)$ we use $\left|\nabla \chi_{1}\right| \leqslant\left|\nabla \chi_{2}\right|+|\nabla \widehat{\chi}|$ combined with the estimate

$$
|\nabla \widehat{\chi}| \leqslant\left|x^{2} \sinh 2 x^{1}\right|+\left|\frac{\partial h\left(x^{1}\right)}{\partial x^{1}}\right|+2 \cosh ^{2} x^{1},
$$

a consequence of the definition (5.9) of $\widehat{\chi}$. So we may use the same arguments as for the proof of $E\left(\mathbf{j}_{2} ; \rho_{2}\right)<\infty$ to conclude $E\left(\mathbf{j}_{1} ; \rho_{1}\right)<\infty$. By (2.14) this proves the claim $T(\psi)<\infty$.

\section{B. No solution to an example with $N=2$ and $\nabla \times \mathrm{v} \neq 0$.}

For the two-body case $(N=2)$ we provide an example with $\nabla \times \mathbf{v} \neq 0$, for which there is no continuously differentiable solution to the problem. This example originated out of discussions with Bröcker [10]. Another, older example is by Taut, Machon, and Eschrig [11].

Example $1(N=2)$. Consider the choice

$$
\begin{aligned}
& \rho(\mathbf{x})=\frac{2}{\pi^{3 / 2}} e^{-\mathbf{x}^{2}} \\
& \mathbf{j}(\mathbf{x})=\frac{\pi^{3 / 2}}{2}\left(0,-2 x^{1} x^{3},-x^{1} x^{2}\right) e^{-\mathbf{x}^{2}},
\end{aligned}
$$

with resulting velocity field

$$
\mathbf{v}(\mathbf{x})=\left(0,-2 x^{1} x^{3},-x^{1} x^{2}\right) .
$$

Clearly $E(\rho)<\infty$ and $E(\mathbf{j}, \rho)<\infty$.

Proposition $1(N=2)$. There exists no solution to the problem with continuously differentiable $\rho_{k} / \rho$ and $\chi_{k}, k=$ 1,2 , when $\rho$ and $\mathbf{j}$ are of the form (5.16).

Remark 1. We have not been able to show that there is no solution $\rho_{1}, \mathbf{j}_{1}, \rho_{2}$, and $\mathbf{j}_{2}$ to the problem, when the solution is only required to satisfy $T(\psi)=E\left(\rho_{1}\right)+E\left(\mathbf{j}_{1}, \rho_{1},\right)+E\left(\rho_{2}\right)+$ $E\left(\mathbf{j}_{2}, \rho_{2}\right)<\infty$, which means less smoothness for $\rho_{1(2)}, \mathbf{j}_{1(2)}$, and $\mathbf{v}_{1(2)}$.

Proof. Introduce the harmonic function on $\mathbb{R}^{3}$,

$$
h\left(x^{1}, x^{2}, x^{3}\right)=\frac{1}{2}\left[\left(x^{1}\right)^{2}+\left(x^{2}\right)^{2}-2\left(x^{3}\right)^{2}\right] .
$$

An easy calculation shows that the curl of $\mathbf{v}$ equals the gradient of $h$,

$$
\nabla \times \mathbf{v}(\mathbf{x})=\nabla h(\mathbf{x})=\left(x^{1}, x^{2},-2 x^{3}\right) .
$$

With the notation and discussion in the previous subsection, in particular in connection with the first relation in Eq. (5.8), we have to look for solutions $\eta_{1}$ and $\widehat{\chi}$ to the relation

$$
\nabla \eta_{1} \times \nabla \hat{\chi}=\nabla h .
$$

But now we claim there are no solutions to (5.20). Indeed, there is even a stronger result due to Bröcker [10], which reads as follows.

Lemma 3. Given the function $h$ (5.18), there are no continuous vector fields $\mathbf{a}$ and $\mathbf{b}$ on $\mathbb{R}^{3}$ with

$$
\mathbf{a} \times \mathbf{b}=\nabla h .
$$

Proof. Assume to the contrary that there are solutions a and b to (5.21). Note that the vector field $\nabla h(\mathbf{x})$ is nonvanishing for $\mathbf{x} \neq 0$. Hence the vector fields $\mathbf{a}$ and $\mathbf{b}$ necessarily share the same property and in addition we must have

$$
\mathbf{a}(\mathbf{x}) \perp \nabla h(\mathbf{x}), \quad \mathbf{x} \neq 0
$$


[and similarly for $\mathbf{b}(\mathbf{x})$ ]. Condition (5.22) written out for $\mathbf{a}(\mathbf{x})=$ $\left(a^{1}(\mathbf{x}), a^{2}(\mathbf{x}), a^{3}(\mathbf{x})\right)$ is

$$
a^{1}(\mathbf{x}) x^{1}+a^{2}(\mathbf{x}) x^{2}-2 a^{3}(\mathbf{x}) x^{3}=0 .
$$

Introduce the vector field

$$
\mathbf{u}(\mathbf{x})=\left(a^{1}(\mathbf{x}), a^{2}(\mathbf{x}),-2 a^{3}(\mathbf{x})\right),
$$

which, by the discussion just made, is nonvanishing for $\mathbf{x} \neq 0$. By (5.23) it is orthogonal to the radius vector $\left(x^{1}, x^{2}, x^{3}\right)$. Hence it is tangential to any sphere centered at the origin and nonvanishing everywhere there. But this contradicts the hairy ball theorem of Brouwer [12]. For modern proofs of this theorem see, e.g., [13], IV, 4.4, [14], Chap.4, Sec.7, Corr.11. A proof using simple analytic tools is given in [15], VI, 2.4.

The proposition is now a direct consequence of this lemma and the preceding discussion.

There is an easier direct proof, that there are no continuously differentiable solutions $\mathbf{a}(\mathbf{x})$ and $\mathbf{b}(\mathbf{x})$ to (5.21), which uses a slightly stronger condition. Indeed, make a Taylor expansion and write $\mathbf{a}(\mathbf{x})=\widehat{\mathbf{a}}+A \mathbf{x}+o\left(|x|^{2}\right)$ and similarly $\mathbf{b}(\mathbf{x})=\widehat{\mathbf{b}}+$ $B \mathbf{x}+o\left(|x|^{2}\right)$, where $A$ and $B$ are $3 \times 3$ matrices. Also let $T=$ $\operatorname{diag}(1,1,-2)$, whence $T \mathbf{x}=\nabla h(\mathbf{x})$. But then the condition (5.21) first says $\mathbf{a} \times \mathbf{b}=0$ and $\widehat{\mathbf{a}} \times B \mathbf{x}-\widehat{\mathbf{b}} \times A \mathbf{x}=T \mathbf{x}$. The first condition says that $\widehat{\mathbf{a}}$ and $\widehat{\mathbf{b}}$ are parallel. Now the case $\widehat{\mathbf{a}}=$ $\widehat{\mathbf{b}}=0$ can be excluded immediately and so we may assume that at least one vector is nonvanishing, say $\widehat{\mathbf{a}} \neq 0$, and that $\widehat{\mathbf{b}}=\lambda \widehat{\mathbf{a}}$. But with $\mathbf{y}=T \mathbf{x}$ this leads to the relation

$$
\widehat{\mathbf{a}} \times(-\lambda A+B) T^{-1} \mathbf{y}=\mathbf{y},
$$

valid for all small $\mathbf{y}$ and hence, by linearity, for all $\mathbf{y}$. In particular this means that $\widehat{\mathbf{a}}$ is orthogonal to all $\mathbf{y}, \widehat{\mathbf{a}} \perp \mathbf{y}$, which is a contradiction.

\section{ACKNOWLEDGMENTS}

We thank E. K. U. Gross for making us aware of this problem and for his encouragement. We are also grateful to Th. Bröcker, O. Lazarev, and V. Rutherfoord for helpful discussions and suggestions, and we thank S. Kvaal for valuable comments on a first draft of this paper. E.H.L. thanks the Simons Foundation for support through Grant No. 230207 and the US National Science Foundation for partial support through Grants No. PHY-0965859 and No. PHY-1265118.

\section{APPENDIX A: PROOF OF LEMMA 1}

With $\kappa_{3}$ as given in Eq. (4.13), $\widehat{\chi}_{3}=\kappa_{3}+h_{3}$ obviously solves the first relation in Eq. (4.10). Inserting this into the two other equations in Eq. (4.10) gives the equations

$$
\begin{aligned}
& \partial_{3}\left(\partial_{1} \eta_{1} \widehat{\chi}_{1}+\partial_{1} \eta_{2} \widehat{\chi}_{2}\right)=\widehat{w}_{2}, \\
& \partial_{2}\left(\partial_{1} \eta_{1} \widehat{\chi}_{1}+\partial_{1} \eta_{2} \widehat{\chi}_{2}\right)=\widehat{w}_{3},
\end{aligned}
$$

with

$$
\begin{aligned}
& \widehat{w}_{2}(\mathbf{x})=-w_{2}(\mathbf{x})-\frac{\partial_{1} \eta_{3}\left(x^{1}, x^{2}\right)}{\partial_{2} \eta_{3}\left(x^{1}, x^{2}\right)} w_{1}(\mathbf{x}), \\
& \widehat{w}_{3}(\mathbf{x})=w_{3}(\mathbf{x})-\partial_{1} \eta_{3}\left(x^{1}, x^{2}\right) \int_{0}^{x^{3}}\left(\partial_{2} \frac{w_{1}}{\partial_{2} \eta_{3}}\right)\left(x^{1}, x^{2}, s\right) d s
\end{aligned}
$$

$$
\begin{aligned}
& +\partial_{2} \eta_{3}\left(x^{1}, x^{2}\right) \int_{0}^{x^{3}}\left(\partial_{1} \frac{w_{1}}{\partial_{2} \eta_{3}}\right)\left(x^{1}, x^{2}, s\right) d s \\
& +\partial_{2} \eta_{3}\left(x^{1}, x^{2}\right) \partial_{1} h_{3}\left(x^{1}\right) .
\end{aligned}
$$

Using the fact that $\mathbf{w}$ has vanishing divergence by its very definition, a short calculation shows that the following necessary and sufficient condition for solving (A1),

$$
\partial_{2} \widehat{w}_{2}(\mathbf{x})=\partial_{3} \widehat{w}_{3}(\mathbf{x}),
$$

is valid for any choice of $h_{3}\left(x^{1}\right)$. So since (A3) holds for each $x^{1},\left(\widehat{w}_{3}, \widehat{w}_{2}\right)$ is a two-dimensional gradient field. In other words there exists $\widehat{w}$ such that $\left(\widehat{w}_{3}, \widehat{w}_{2}\right)=\left(\partial_{2} \widehat{w}, \partial_{3} \widehat{w}\right)$ holds. $\widehat{w}$ can be obtained by integrating this vector field, for example, from $\left(x^{1}, 0,0\right)$ - with arbitrary initial value $\widehat{h}_{1}\left(x^{1}\right)$ - to $\left(x^{1}, x^{2}, 0\right)$ and from there to $\left(x^{1}, x^{2}, x^{3}\right)$. Thus

$$
\begin{aligned}
\widehat{w}(\mathbf{x})= & \int_{0}^{x^{2}} \widehat{w}_{3}\left(x^{1}, s, x^{3}=0\right) d s \\
& +\int_{0}^{x^{3}} \widehat{w}_{2}\left(x^{1}, x^{2}, t\right) d t+\widehat{h}_{1}\left(x^{1}\right) .
\end{aligned}
$$

So with our choice (4.13)-(4.15) for $\widehat{\chi}_{1}, \widehat{\chi}_{2}$, and $\widehat{\chi}_{3}$ and the choice $\widehat{h}_{1}\left(x^{1}\right)=\partial_{1} \eta_{1}\left(x^{1}\right) h_{1}\left(x^{1}\right)$, the relation

$$
\partial_{1} \eta_{1} \widehat{\chi}_{1}+\partial_{1} \eta_{2} \widehat{\chi}_{2}=\widehat{w}
$$

is satisfied. Observe that

$$
\widehat{w}_{3}\left(x^{1}, x^{2}, 0\right)=w_{3}\left(x^{1}, x^{2}, 0\right)+\partial_{2} \eta_{3}\left(x^{1}, x^{2}\right) \partial_{1} h_{3}\left(x^{1}\right)
$$

holds. Therefore also relation (4.10) is valid and the proof of Lemma 1 is complete.

\section{APPENDIX B: PROOF OF LEMMA 2}

We start with estimates for $\nabla \kappa_{3}$. By (4.13),

$$
\begin{aligned}
\partial_{1} \kappa_{3}(\mathbf{x})= & -\frac{\partial_{1} \partial_{2} \eta_{3}\left(x^{1}, x^{2}\right)}{\left[\partial_{2} \eta_{3}\left(x^{1}, x^{2}\right)\right]^{2}} \int_{0}^{x^{3}} w_{1}\left(x^{1}, x^{2}, s\right) d s \\
& +\frac{1}{\partial_{2} \eta_{3}\left(x^{1}, x^{2}\right)} \int_{0}^{x^{3}} \partial_{1} w_{1}\left(x^{1}, x^{2}, s\right) d s, \\
\partial_{2} \kappa_{3}(\mathbf{x})= & -\frac{\partial_{2}^{2} \eta_{3}\left(x^{1}, x^{2}\right)}{\left[\partial_{2} \eta_{3}\left(x^{1}, x^{2}\right)\right] 2} \int_{0}^{x^{3}} w_{1}\left(x^{1}, x^{2}, s\right) d s \\
& +\frac{1}{\partial_{2} \eta_{3}\left(x^{1}, x^{2}\right)} \int_{0}^{x^{3}} \partial_{2} w_{1}\left(x^{1}, x^{2}, s\right) d s, \\
\partial_{3} \kappa_{3}(\mathbf{x})= & \frac{1}{\partial_{2} \eta_{3}\left(x^{1}, x^{2}\right)} w_{1}(\mathbf{x}) .
\end{aligned}
$$

To see that all $\left|\partial_{j} \kappa_{3}(\mathbf{x})\right|, j=1,2,3$, are bounded, we proceed as follows. Let $W$ stand for any of the quantities $w_{1}, \partial_{1} w_{1}$, or $\partial_{2} w_{1}$. By the assumption (4.1) there exists a constant $0<$ $C_{1}<\infty$ such that

$$
\begin{aligned}
|W(\mathbf{x})| \leqslant & C_{1}\left[1+\left(x^{1}\right)^{2}\right]^{-(1+\delta) / 2}\left[1+\left(x^{2}\right)^{2}\right]^{-(1+\delta) / 2} \\
& \times\left[1+\left(x^{3}\right)^{2}\right]^{-(1+\delta) / 2}
\end{aligned}
$$


holds. Therefore there is another constant $0<C_{2}<\infty$, such that the bound

$$
\left|\int_{0}^{x^{3}} W\left(x^{1}, x^{2}, s\right) d s\right| \leqslant \int_{-\infty}^{\infty}\left|W\left(x^{1}, x^{2}, s\right)\right| d s \leqslant C_{2}\left[1+\left(x^{1}\right)^{2}\right]^{-(1+\delta) / 2}\left[1+\left(x^{2}\right)^{2}\right]^{-(1+\delta) / 2}
$$

is valid. With this preparation we start with an estimate for the first term contributing to $\partial_{1} \kappa_{3}$, which we call $A_{1}$. Now by (4.12),

$$
\left|\frac{\partial_{1} \partial_{2} \eta_{3}\left(x^{1}, x^{2}\right)}{\left[\partial_{2} \eta_{3}\left(x^{1}, x^{2}\right)\right] 2}\right|=\frac{N-2}{N} \frac{\left[1+\left(x^{1}+\alpha\right)^{2}\right]^{-(1+\delta) / 2}\left[1+\left(x^{2}+\gamma\right)^{2}\right]^{(1+\delta) / 2}}{\left[1-\eta_{1}(\mathbf{x})-\eta_{2}(\mathbf{x})\right]^{2}} \leqslant C_{3}\left[1+\left(x^{2}+\gamma\right)^{2}\right]^{(1+\delta) / 2}
$$

for yet another finite constant $C_{3}$. We have used the relation

$$
\partial_{1} \partial_{2} \eta_{3}\left(x^{1}, x^{2}\right)=-\frac{4}{m^{2} N(N-2)\left[1+\left(x^{1}+\alpha\right)^{2}\right]^{(1+\delta) / 2}\left[1+\left(x^{2}+\gamma\right)^{2}\right]^{(1+\delta) / 2}} .
$$

Combining this estimate with the estimate (B3) for the choice $W=w_{1}$ and with the estimate

$$
\sup _{x^{2} \in \mathbb{R}}\left[1+\left(x^{2}+\gamma\right)^{2}\right]^{(1+\delta) / 2}\left[1+\left(x^{2}\right)^{2}\right]^{-(1+\delta) / 2}<\infty
$$

shows that $A_{1}$ is bounded.

As for the second contribution to $\partial_{1} \kappa_{3}$, and which we call $A_{2}$, we use the estimate (B3) for $W=\partial_{1} w_{1}$ combined with

$$
\left|\frac{1}{\partial_{2} \eta_{3}\left(x^{1}, x^{2}\right)}\right| \leqslant C_{4}\left[1+\left(x^{2}+\gamma\right)^{2}\right]^{(1+\delta) / 2},
$$

which follows from (4.11) and (4.12), and the estimate (B5) to conclude that $A_{2}$ is also bounded.

We turn to an estimate for $\partial_{2} \kappa_{3}$ and start with the first contribution, which we call $B_{1}$. The relation

$$
\begin{aligned}
\partial_{2}^{2} \eta_{3}(\mathbf{x})= & -\frac{2(1+\delta)\left(x^{2}+\gamma\right)}{m(N-2)\left(1+\left(x^{2}+\gamma\right)^{2}\right)^{(3+\delta) / 2}} \\
& \times\left[1-\eta_{1}(\mathbf{x})-\eta_{2}(\mathbf{x})\right]
\end{aligned}
$$

gives the estimate

$$
\begin{aligned}
& \left|\frac{\partial_{2}^{2} \eta_{3}\left(x^{1}, x^{2}\right)}{\left[\partial_{2} \eta_{3}\left(x^{1}, x^{2}\right)\right]^{2}}\right| \\
& \quad=\frac{(1+\delta) m(N-2)}{2} \frac{\left|x^{2}+\gamma\right|\left[1+\left(x^{2}+\gamma\right)^{2}\right]^{-(1-\delta) / 2}}{\left[1-\eta_{1}(\mathbf{x})-\eta_{2}(\mathbf{x})\right]} \\
& \quad \leqslant C_{5}\left[1+\left(x^{2}+\gamma\right)^{2}\right]^{\delta / 2} \\
& \quad \leqslant C_{5}\left[1+\left(x^{2}+\gamma\right)^{2}\right]^{(1+\delta) / 2}
\end{aligned}
$$

Again we have used (4.12) and the trivial bound

$$
\left|x^{2}+\gamma\right|\left[1+\left(x^{2}+\gamma\right)^{2}\right]^{-1} \leqslant 1 .
$$

We combine this bound with the bound (B3) for the choice $W=w_{1}$ and the bound (B5) to conclude that $B_{1}$ is bounded.

As for the second contribution to $\partial_{2} \kappa_{3}$ and which we call $B_{2}$, we proceed in analogy to the proof of the estimate of $A_{2}$. That is, we use (B6) and (B5) and (B3) for the choice $W=\partial_{2} w_{1}$ to conclude that $B_{2}$ is bounded.

Finally we use (B2) for the choice $W-w_{1}$ and (B6) to conclude that $\left|\partial_{3} \kappa_{3}\right|$ is bounded.
As for $\kappa_{1}$, we start with

$$
\left|\kappa_{1}(\mathbf{x})\right| \leqslant \sum_{j=1}^{3}\left|\kappa_{1, j}(\mathbf{x})\right| .
$$

The boundedness of $\left|\nabla \kappa_{1,1}\right|$, see (4.14), follows similar to one for $\left|\nabla \kappa_{3}\right|$. Due to the presence of the factor $\partial_{1} h_{3}\left(x^{1}\right), \kappa_{1,2}(\mathbf{x})$ is smooth, vanishes for all large $x^{1}$, and has bounded derivatives; that is, $\left|\nabla \kappa_{1,2}\right|$ is bounded. By the definition of $\kappa_{1,3}$ it remains to estimate

$$
\begin{aligned}
\partial_{1} \kappa_{1,3}(\mathbf{x})= & -\left(\partial_{1} \frac{1}{\partial_{1} \eta_{1}}(\mathbf{x})\right)\left[\left(\partial_{1} \eta_{2}\right) h_{2}\right](\mathbf{x}) \\
& -\frac{1}{\partial_{1} \eta_{1}(\mathbf{x})}\left[\left(\partial_{1}^{2} \eta_{2}\right) h_{2}\right](\mathbf{x}) \\
& -\frac{1}{\partial_{1} \eta_{1}(\mathbf{x})}\left[\left(\partial_{1} \eta_{2}\right) \partial_{1} h_{2}\right](\mathbf{x})
\end{aligned}
$$

since $\kappa_{1,3}$ is a function of $x^{1}$ only. The relations

$$
\begin{aligned}
\partial_{1} \frac{1}{\partial_{1} \eta_{1}}(\mathbf{x})= & m N(1+\delta) x^{1}\left[1+\left(x^{1}\right)^{2}\right]^{(\delta-1) / 2} \\
\partial_{1} \eta_{2}(\mathbf{x})= & \frac{1}{m(N-1)}\left[1+\left(x^{1}+\beta\right)^{2}\right]^{-(1+\delta) / 2}\left[1-\eta_{1}(\mathbf{x})\right] \\
& -\frac{1}{m N(N-1)} \xi\left(x^{1}+\beta\right)\left[1+\left(x^{1}+\beta\right)^{2}\right]^{-(1+\delta) / 2}
\end{aligned}
$$

show that

$$
\left(\partial_{1} \frac{1}{\partial_{1} \eta_{1}}\right) \partial_{1} \eta_{2}
$$

is bounded. Since $h_{2}$ is bounded this shows that the first term on the right-hand side of (B8) is bounded. By calculating $\partial_{1}^{2} \eta_{2}$, a similar argument shows that

$$
\frac{1}{\partial_{1} \eta_{1}} \partial_{1}^{2} \eta_{2}
$$

is bounded, such that the second term on the right-hand side of (B8) is also bounded. The third term is bounded, since $\partial_{1} h_{2}\left(x^{1}\right)$ vanishes for all large $x^{1}$. In conclusion, we have established that $\left|\nabla \kappa_{1,3}\right|=\left|\partial_{1} \kappa_{1,3}\right|$ is bounded and this completes the proof of Lemma 2. 
[1] S. K. Ghosh and A. K. Dhara, Phys. Rev. A 38, 1149 (1988).

[2] E. K. U. Gross, in Density Functional Theory, edited by R. F. Nalewajski, Topics in Current Chemistry (Springer, Berlin/Heidelberg, 1996), Vol. 181, p. 81.

[3] J. E. Harriman, Phys. Rev. A 24, 680 (1981).

[4] E. H. Lieb, in Physics as Natural Philosophy: Essays in Honor of Laszlo Tisza on His 75th Birthday, edited by A. Shimony and H. Feshbach (MIT Press, Cambridge, MA, 1982), pp. 111-149; Int. J. Quantum Chem. 24, 243 (1983); a further revision appears in Density Functional Methods in Physics, edited by R. Dreizler and J. da Providencia, Nato ASI Series (Plenum, New York, 1985), Vol. 123, p. 31.

[5] G. Zumbach, Phys. Rev. A 31, 1922 (1985).

[6] O. Lazarev and E. H. Lieb, arXiv:1205.5059 [Indiana Univ. Math. J. (to be published)].
[7] V. Rutherfoord, arXiv:1212.5759.

[8] C. R. Hobby and J. R. Rice, Proc. Am. Math. Soc. 16, 665 (1965).

[9] A. Pincus, Proc. Am. Math. Soc. 60, 82 (1976).

[10] Th. Bröcker (private communication).

[11] M. Taut, P. Machon, and H. Eschrig, Phys. Rev. A 80, 022517 (2009).

[12] L. E. J. Brouwer, Math. Ann. 71, 97 (1911).

[13] A. Dold, Lectures on Algebraic Topology, 2nd. ed. (Springer, Berlin, 1980).

[14] E. H. Spanier, Algebraic Topology (McGraw-Hill, New York, 1981).

[15] Th. Bröcker, Analysis III, http://www.mathematik.uniregensburg.de/broecker/index.htm. 